\title{
Características diferenciales de la hepatitis autoinmune en adultos mayores colombianos: estudio de cohorte
}

\section{Differential characteristics of autoimmune hepatitis in Colombian older adults: a cohort study}

\author{
Gabriel Sebastián Díaz-Ramírez, ${ }^{*}$ Omar Yesid Martínez-Casas, ${ }^{1}$ Juan Ignacio Marín-Zuluaga, ${ }^{2}$ Jorge Hernando Donado-Gómez, ${ }^{3}$ \\ Octavio Muñoz-Maya, ${ }^{2}$ Óscar Santos-Sánchez, ${ }^{2}$ Juan Carlos Restrepo-Gutiérrez. ${ }^{2}$
}

\footnotetext{
Hepatología clínica, Universidad de Antioquia, Medellín, Colombia.

2 Grupo Gastro-hepatología, Universidad de Antioquia. Unidad de hepatología y trasplante hepático, Hospital Pablo Tobón Uribe. Medellín, Colombia.

3 Grupo Gastro-hepatología, Universidad de Antioquia. Unidad de epidemiología, Hospital Pablo Tobón Uribe. Medellín, Colombia.
}

*Correspondencia: sebastiandiazr@gmail.com

\begin{abstract}
Resumen
Introducción: en la hepatitis autoinmune ( $\mathrm{HAl})$, los ancianos representan una población especial debido a que, según se ha descrito, poseen una predisposición mediada por el antígeno leucocitario humano (Human Leukocyte Antigen, HLA) y padecen un curso indolente y progresivo de la enfermedad. No se conocen datos en la población latinoamericana. Objetivos: Analizar comparativamente las características de los pacientes con HAl mayores y menores de 65 años en cuanto a su presentación clínica, diagnóstico, tratamiento, respuesta al tratamiento y curso de la enfermedad. Métodos: estudio de cohorte retrospectiva de pacientes con HAl evaluados entre enero de 2010 y diciembre de 2016. Se realizó un análisis estadístico utilizando el programa SPSS versión 20.1. Resultados: se incluyeron 214 pacientes. Los pacientes ancianos tuvieron, en su mayoría, hipertensión arterial (34,5\% frente a $15,1 \%, p=0,011)$, dislipidemia $(20,7 \%$ frente a 5,9\%, $p=$ $0,006)$ y enfermedad cardiovascular $(17,2 \%$ frente a $2,7 \%, p=0,001)$. Además, al momento del diagnóstico, los pacientes ancianos tuvieron una mayor frecuencia de cirrosis evaluada histológica y radiológicamente $(55,1 \%$ frente a $33,5 \%, p=0,024)$. Los pacientes mayores tuvieron una mayor remisión bioquímica con el tratamiento (100\% frente a 83,9\%,p=0,022). No hubo diferencias en la analítica hepática, autoanticuerpos, tipo de tratamiento farmacológico recibido, recaídas, efectos adversos relacionados con el tratamiento, requerimiento de trasplante hepático y muerte. Conclusión: La HAl afecta a población colombiana adulta de todas las edades y debe ser considerada en el abordaje diagnóstico de los ancianos con enfermedad hepática, debido a que este grupo de pacientes tiene una mayor frecuencia de cirrosis al momento del diagnóstico. Un diagnóstico temprano es importante debido a que el tratamiento es efectivo y bien tolerado.
\end{abstract}

\section{Palabras clave}

Hepatitis autoinmune, Latinoamérica, ancianos.

\begin{abstract}
Introduction: Elderly patients with autoimmune hepatitis $(\mathrm{AlH})$ are a special population because of predisposition mediated by the human leukocyte antigen (HLA) system. An indolent and progressive course of the disease has been described. No data are known for the Latin American population. Objectives: This study compares clinical presentations, diagnoses, treatments, responses to treatment and course of disease for AlH patients who are over 65 years of age with those of AlH patients who are under 65. Methods: This is a retrospective cohort study of patients with HAl evaluated between January 2010 and December 2016. Statistical analyses used SPSS version 20.1. Results: Two hundred fourteen patients were included. Elderly patients had hypertension $(34.5 \%$ vs $15.1 \%, p=0.011)$, dyslipidemia $(20.7 \%$ vs $5.9 \%, p=0.006)$ and cardiovascular disease $(17.2 \%$ vs $2.7 \%, p=$ 0.001 ) more frequently than did the younger patients. In addition, the elderly had a higher frequency of cirrhosis confirmed histologically and radiologically $(55.1 \%$ vs. $33.5 \%, p=0.024)$ at the time of diagnosis. Older patients had a higher rate of biochemical remission resulting from treatment $(100 \%$ vs $83.9 \%, p=0.022)$. There were no differences in hepatic analyses, autoantibodies, type of pharmacological treatment received, relapses, adverse effects related to treatment, requirements for liver transplantation and deaths. Conclusion: $\mathrm{AlH}$ affects the Colombian adult population at all ages and should be considered in the diagnostic approach of elderly patients who have liver disease because this group has a higher frequency of cirrhosis at the time of diagnosis. Early diagnosis is important because treatment is effective and well tolerated.
\end{abstract}

Keywords

Autoimmune hepatitis, Latin America, elderly patients. 


\section{INTRODUCCIÓN}

Desde su primera descripción en 1950, la hepatitis autoinmune (HAI) fue considerada como una enfermedad de mujeres jóvenes (1); sin embargo, en estudios posteriores se demostró un comportamiento bimodal, con picos de ocurrencia entre los 10-30 años y entre los 40-50 años (2), aunque puede afectar a personas de todos los grupos de edad (3-5). La HAI es una causa importante de morbilidad y mortalidad, así como de falla hepática aguda, cirrosis hepática, de requerimiento de trasplante hepático y de disfunción hepática postrasplante (6).

Los adultos mayores representan una población especial en la HAI por varias razones (7): primero, existen diferencias entre los estudios en cuanto al punto de corte de la edad para considerar como adulto mayor a un paciente (diagnóstico a partir de los 60 o 65 años); segundo, se describe una posible predisposición genética en este grupo de edad debido a una mayor prevalencia de HLA-DR4 y HLA-DRB1*04 (5, $8)$; tercero, constituyen un reto diagnóstico para el médico debido a que se diagnóstica de manera tardía en comparación con la población más joven (9), y existe una frecuencia mayor de pacientes asintomáticos y cirróticos al momento del diagnóstico $(5,8,10)$. En cuanto a la respuesta al tratamiento inmunosupresor, se han reportado tasas de remisión similares con menos recaídas después del retiro del tratamiento (5), aunque con una mayor frecuencia de intolerancia y efectos adversos relacionados con este $(10,11)$. El curso de la HAI en población mayor suele ser indolente y progresivo, y puede enmascararse por la presencia de otras enfermedades, especialmente las autoinmunes (11).

La información existente sobre el comportamiento diferencial de la HAI en adultos mayores proviene de estudios retrospectivos realizados en población norteamericana (8), europea $(4,10,12-14)$ y asiática (15), además de revisiones sistemáticas de la literatura (5). En Latinoamérica, se desconocen datos en este grupo de pacientes.

El objetivo de este estudio es evaluar de manera comparativa las características diferenciales, tanto clínicas como diagnósticas, de tratamiento y respuesta al mismo, así como curso y pronóstico de la HAI entre los pacientes menores y mayores de 65 años.

\section{MATERIALES Y MÉTODOS}

\section{Diseño y muestra}

Este fue un estudio retrospectivo de cohorte histórica. Se realizó un muestreo basado en el diagnóstico de la HAI (código K754), según la Clasificación Internacional de Enfermedades (CIE 10), en los registros de historia clí- nica de los pacientes atendidos en los servicios de urgencias, hospitalización y consulta externa del Hospital Pablo Tobón Uribe (HPTU) de Medellín, Colombia durante el período comprendido entre enero de 2010 a diciembre de 2016. No se estimó un tamaño de muestra debido a que se incluyeron todos los pacientes con diagnóstico de HAI atendidos durante el período del estudio.

\section{Población}

Se incluyeron pacientes con diagnóstico de HAI a partir delos 16 años, que cumplieran con los criterios diagnósticos simplificados publicados en el 2008 por el Grupo Internacional de Hepatitis Autoinmune (GIHA) (16), y aquellos con puntaje $<6$, en los cuales la respuesta al tratamiento farmacológico ayudara a confirmar el diagnóstico $(2,17)$.

Se excluyeron los pacientes diagnosticados antes de los 16 años, aquellos con síndromes de superposición de hepatitis autoinmune-colangitis biliar primaria (HAI-CBP) $\mathrm{y}$ hepatitis autoinmune-colangitis esclerosante primaria (HAI-CEP), pacientes con falla hepática aguda por HAI y con HAI inducida por medicamentos. Asimismo, se excluyeron pacientes con ausencia de datos clínicos, bioquímicos o histológicos, lo cual no permite un diagnóstico adecuado.

\section{Variables}

Se realizó una revisión de los registros electrónicos de historia clínica del hospital y se recolectaron los datos desde el diagnóstico de HAI hasta el último seguimiento clínico mediante un instrumento de recolección previamente diseñado.

Se recolectaron variables sociodemográficas (edad en el momento del diagnóstico de HAI, sexo, raza), presencia de comorbilidades, incluyendo otras enfermedades autoinmunes y variables clínicas, como la forma de presentación, la cual se clasificó así: asintomáticos, aquellos con una alteración bioquímica hepática sin síntomas; con síntomas inespecíficos, aquellos con una alteración bioquímica hepática y con presencia de síntomas, como astenia, hiporexia y fiebre; con hepatitis aguda, aquellos con dolor abdominal, náuseas, fiebre e ictericia asociada a una elevación de las transaminasas al menos 3 veces el límite superior de normalidad, sin cumplir los criterios de falla hepática aguda; $y$ con cirrosis hepática, aquellos diagnosticados por biopsia clínica o imaginológicamente.

En cuanto a las variables de laboratorio, se incluyeron los niveles de aspartato aminotransferasa (AST), alanina aminotransferasa (ALT), bilirrubina total, fosfatasa alcalina, albúmina sérica, inmunoglobulina $\mathrm{G}$ ( $\operatorname{IgG}$ ) sérica, tiempo de protrombina e International Normalized Ratio (INR) al momento del diagnóstico y durante el seguimiento, para 
evaluar la respuesta al tratamiento. Además, se describen los títulos de autoanticuerpos (antinucleares, antimúsculo liso y antimitocondriales).

Las características histológicas se clasificaron según las recomendaciones para el diagnóstico de HAI descritas por el GIHA (16), como: típica de HAI, ante la presencia de hepatitis de interfase, infiltrados linfocítico/plasmocitario en espacios porta con extensión al lobulillo, emperipolesis y formación de rosetas; compatible con HAI, es decir, hepatitis crónica con infiltrado linfocítico sin los otros hallazgos típicos de la hepatitis autoinmune. El grado de fibrosis hepática se evaluó según la escala METAVIR, grados F0 a F4, donde F0 representa la ausencia de fibrosis y $\mathrm{F} 4$, fibrosis avanzada con cirrosis.

Se evaluó el tipo de tratamiento farmacológico de inducción y de mantenimiento instaurado. El esquema de tratamiento usado por el grupo de hepatología se describe a continuación: durante la fase de inducción se administró prednisolona $0,5-1 \mathrm{mg} / \mathrm{kg} /$ día y azatioprina $1 \mathrm{mg} / \mathrm{kg} /$ día; posteriormente, hubo un descenso gradual de la dosis de prednisolona en los siguientes tres meses y aumento progresivo de la dosis de azatioprina hasta de $2 \mathrm{mg} / \mathrm{kg} /$ día, según la tolerancia y la respuesta al tratamiento durante la fase de mantenimiento. En la evaluación de la respuesta al tratamiento se establecieron las siguientes categorías: remisión bioquímica, a la normalización de transaminasas e IgG; respuesta parcial, a la mejoría clínica y de transaminasas, pero sin normalización de estas; falla terapéutica, no lograr una disminución de al menos el $25 \%$ de los niveles de transaminasas con respecto al valor en el inicio del tratamiento; recaída, una nueva elevación de ALT > 3 veces el límite superior de normalidad (LSN), según los criterios de la GIHA, un aumento de los niveles de IgG o un empeoramiento de los hallazgos histológicos después de haber alcanzado la remisión con el tratamiento farmacológico (2).

Se realizó un seguimiento hasta la última valoración clínica, y durante este se encontraron complicaciones, como el desarrollo de cirrosis (en los que se diagnosticaron no cirróticos), el requerimiento de trasplante hepático (nuestro grupo tiene como criterio considerar esta opción en pacientes $\leq 65$ años y a partir de los 65 años en casos seleccionados), la recurrencia postrasplante, el retrasplante hepático y la muerte.

\section{Aspectos éticos}

El estudio se mantuvo dentro de los parámetros de la Declaración de Helsinki de 2013 para estudios con seres humanos y la reglamentación sobre la investigación clínica en Colombia (resolución 008430 de 1993). Además, fue aprobado por el comité de ética del HPTU. Por último, el manus- crito final se adhirió a las recomendaciones Strengthening the Reporting of Observational Studies in Epidemiology (STROBE) para el reporte de los estudios observacionales (18).

\section{Análisis estadístico}

El análisis estadístico se realizó con el software estadístico SPSS versión 20.1 (SPSS Inc.). Las variables categóricas se presentan en frecuencias absolutas y relativas, las variables continuas como media y desviación estándar según la distribución normal o mediana y el rango intercuartílico (RIC) con una distribución anormal según la prueba de Kolmogórov-Smirnov. Las diferencias entre grupos se establecieron con la prueba $\chi^{2}$ para variables categóricas y la prueba $U$ de Mann-Whitney para la diferencia de medianas. Los valores de $p$ se calcularon a dos colas, donde $p$ $<0,05$ representa una significancia estadística.

\section{RESULTADOS}

En total, 214 pacientes cumplieron los criterios de inclusión, de los cuales 185 y 29 fueron diagnosticados con HAI antes y a partir de los 65 años, respectivamente. Se excluyeron 83 pacientes por múltiples causas (Figura 1). La distribución de edad al momento del diagnóstico de HAI se describe en la Figura 2.

En los dos grupos de edad, la mayoría eran mujeres. Hubo una diferencia estadísticamente significativa en la mediana de seguimiento desde el diagnóstico de HAI de 50 y 19 meses en el grupo de $<65$ años y de $>65$ años, respectivamente, $(p<0,001)$ (Tabla 1).

\section{Aspectos clínicos}

En ambos grupos, la comorbilidad más frecuente fue el hipotiroidismo. No hubo diferencias en cuanto a otras enfermedades autoinmunes. Se encontraron diferencias con significancia estadística en la presencia de hipertensión arterial $(15,1 \%$ frente a $34,5 \%, p=0,011)$, dislipidemia $(5,9 \%$ frente a $20,7 \%, p=0,006)$ y enfermedad cardiovascular $(2,7 \%$ frente a $17,2 \%, p=0,001)$, las cuales fueron frecuentes en los pacientes diagnosticados con HAI a partir de los 65 años. La principal forma de presentación clínica en ambos grupos fue la hepatitis aguda; de estos, el 27,5\% (26,6 \% en los jóvenes y $33,3 \%$ en los mayores) ya estaban cirróticos (hepatitis aguda sobre hígado cirrótico).

En total, $21 \%$ y $31 \%$ de los menores y mayores de 65 años, respectivamente, estaban clínicamente cirróticos al momento del diagnóstico, diferencia que no fue significativa; sin embargo, al evaluar el grado de fibrosis por imagen y biopsia hepática se encontró una mayor frecuencia de 


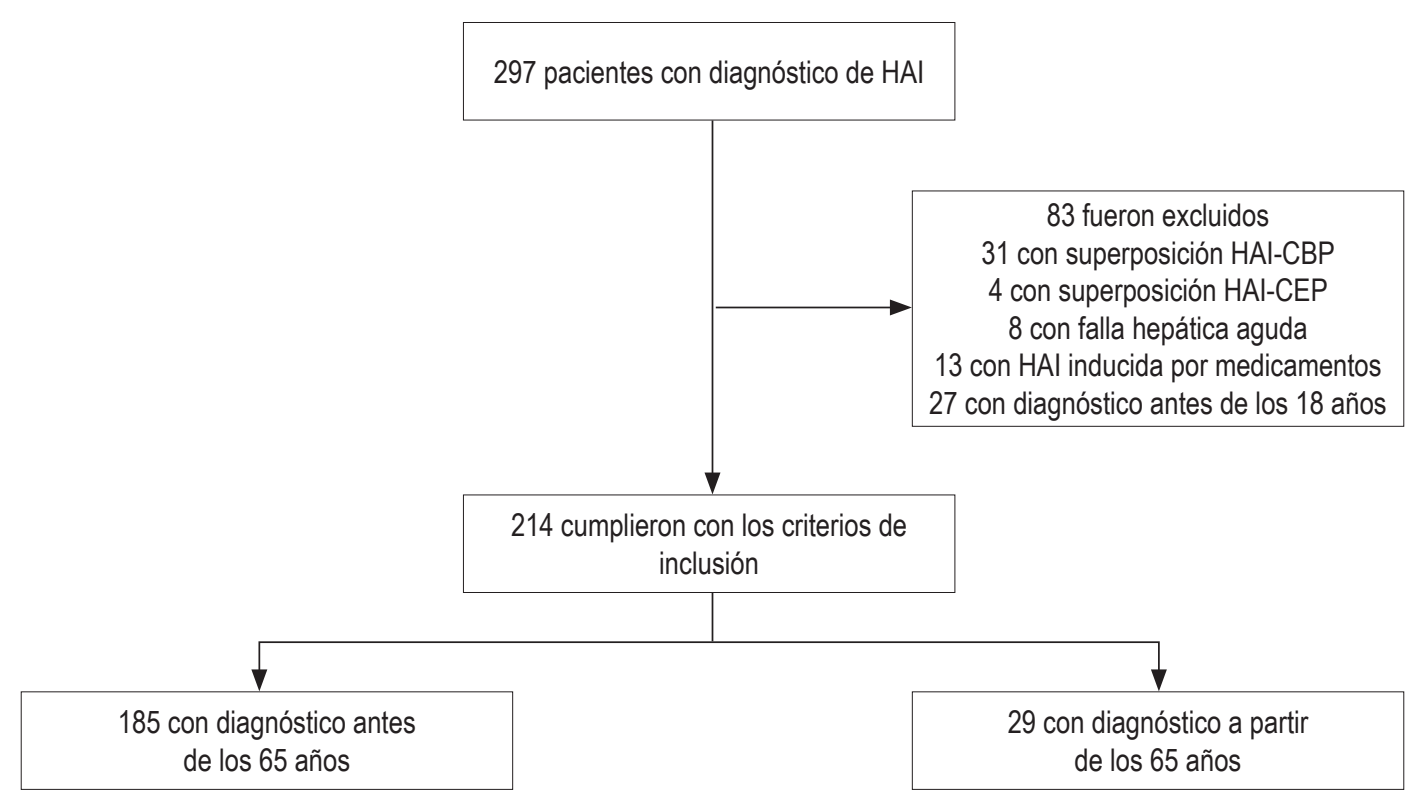

Figura 1. Diagrama de flujo del estudio. HAI: hepatitis autoinmune; HAI-CBP: hepatitis autoinmune-colangitis biliar primaria; HAI-CEP: hepatitis autoinmune-colangitis esclerosante primaria.

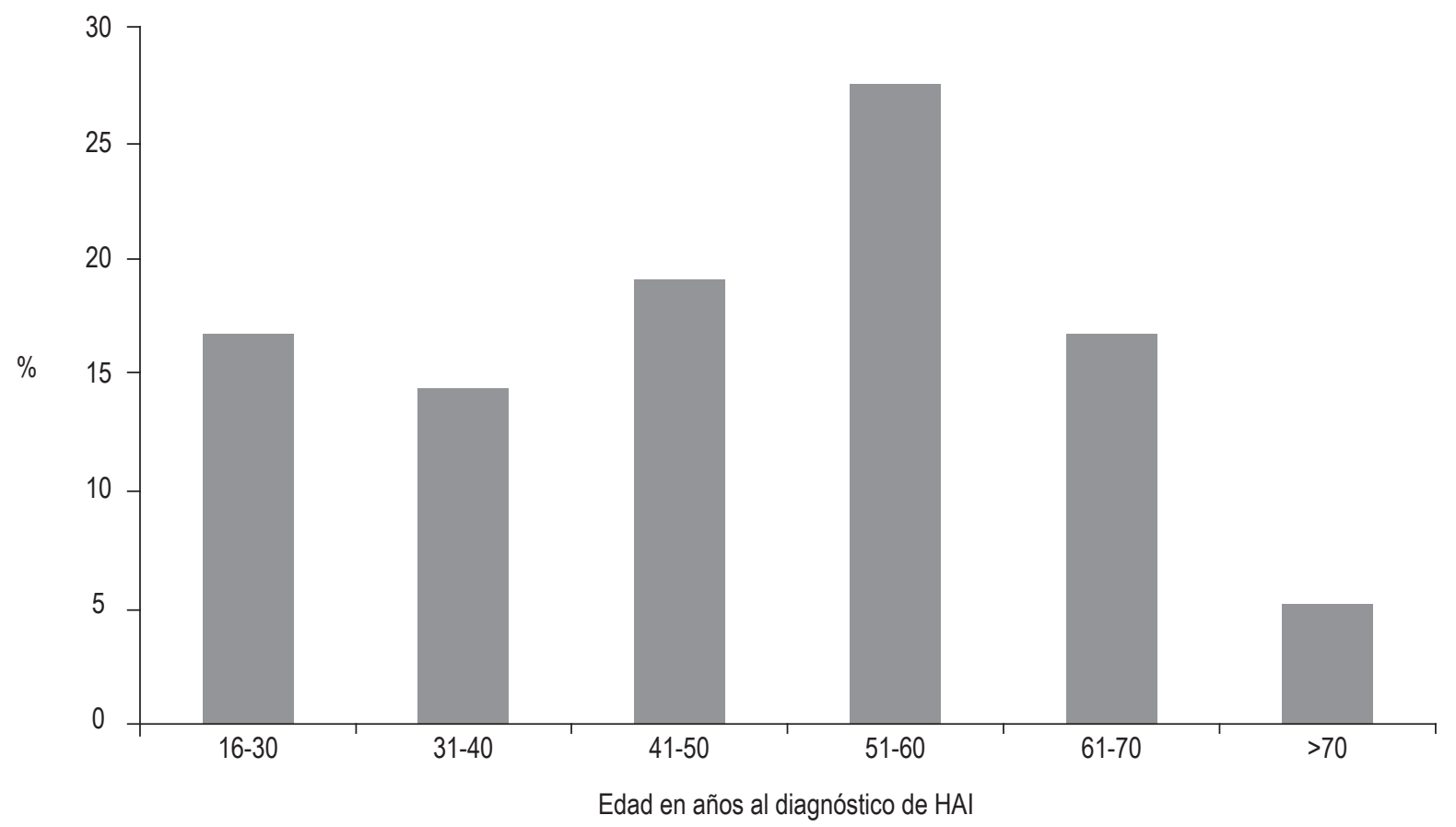

Figura 2. Distribución de la edad al momento del diagnóstico de HAI. 
Tabla 1. Características demográficas, clínicas, serológicas e histológicas de los pacientes con HAI de acuerdo con la edad

\begin{tabular}{|c|c|c|c|}
\hline & $\begin{array}{c}\text { Pacientes jóvenes } \\
<65 \text { años } \\
n=185\end{array}$ & $\begin{array}{c}\text { Pacientes mayores } \\
\begin{array}{c}\geq 65 \text { años } \\
n=29\end{array}\end{array}$ & Valor de $p$ \\
\hline Sexo femenino - $\mathrm{n}(\%)$ & $167(90,3)$ & $28(96,5)$ & 0,269 \\
\hline Edad al diagnóstico, mediana en años (RIC) & $47(35-56)$ & $71(66-74,5)$ & $<0,001$ \\
\hline Tiempo de seguimiento en meses, mediana (RIC) & $50(17-80,5)$ & $19(5,5-37,5)$ & 0,003 \\
\hline Comorbilidad autoinmune - $\mathrm{n}(\%)$ & $56(30,3)$ & $9(31)$ & 0,934 \\
\hline \multicolumn{4}{|l|}{ Comorbilidades - $\mathrm{n}(\%)$} \\
\hline Hipotiroidismo & $50(30,8)$ & $13(44,8)$ & 0,135 \\
\hline Hipertensión arterial & $28(15,1)$ & $10(34,5)$ & 0,011 \\
\hline Dislipidemia & $11(5,9)$ & $6(20,7)$ & 0,006 \\
\hline Enfermedad cardiovascular* & $5(2,7)$ & $5(17,2)$ & 0,001 \\
\hline Diabetes mellitus & $15(8,1)$ & $4(13,8)$ & 0,317 \\
\hline Obesidad & $7(3,8)$ & $0(0)$ & 0,284 \\
\hline Enfermedad renal crónica & $1(0,5)$ & $1(3,4)$ & 0,130 \\
\hline \multicolumn{4}{|l|}{ Forma de presentación clínica - n (\%) } \\
\hline Asintomáticos, alteración bioquímica hepática & $34(18,4)$ & $5(17,2)$ & 0,883 \\
\hline Síntomas inespecíficos & $37(20)$ & $5(17,2)$ & 0,728 \\
\hline Hepatitis aguda & $60(32,4)$ & $9(31)$ & 0,881 \\
\hline Cirrosis hepática & $39(21,1)$ & $9(31)$ & 0,232 \\
\hline Sin dato & $15(8,1)$ & $1(3,5)$ & 0.375 \\
\hline AST, mediana U/L (RIC) & $226(99-718)$ & $313(178-727)$ & 0,770 \\
\hline ALT, mediana U/L (RIC) & $260(95-698)$ & $222(105-705)$ & 0,678 \\
\hline Fosfatasa alcalina, mediana U/L (RIC) & $178(116-297)$ & $170(138-278)$ & 0,827 \\
\hline Niveles de lgG al diagnóstico, mediana mg/dL (RIC) & $2000(1700-2501)$ & $1863(1485-2800)$ & 0,661 \\
\hline ANA positivos $\geq 1: 40-n(\%)$ & $142(76,8)$ & $24(82,8)$ & 0,471 \\
\hline ASMA positivos $\geq 1: 40-n(\%)$ & $57(30,8)$ & $13(44,8)$ & 0,135 \\
\hline AMA positivos - $n(\%)$ & $12(6,4)$ & $0(0)$ & 0,158 \\
\hline Biopsia hepática - n (\%) & $153(82,7)$ & $24(82,8)$ & 0,994 \\
\hline \multicolumn{4}{|l|}{ Fibrosis hepática al momento del diagnóstico - $\mathrm{n}(\%)^{\pi}$} \\
\hline F0-F1 & $18(11,8)$ & $3(12,5)$ & 0,918 \\
\hline F2-F3 & $42(27,4)$ & $5(20,8)$ & 0,495 \\
\hline F4 & $61(39,9)$ & $14(58,3)$ & 0,089 \\
\hline Sin dato & $32(20,9)$ & $2(8,3)$ & 0,146 \\
\hline Cirróticos por clínica, laboratorios, imagen y biopsia - n (\%) & $62(33,5)$ & $16(55,1)$ & 0,024 \\
\hline \multicolumn{4}{|l|}{ Hallazgo en biopsia $-n(\%)^{\varepsilon}$} \\
\hline Compatible con HAl & $48(31,4)$ & $4(16,6)$ & 0,141 \\
\hline Típico de HAl & $105(68,6)$ & $20(83,4)$ & 0,141 \\
\hline \multicolumn{4}{|l|}{ Puntaje diagnóstico de HAI - $\mathrm{n}(\%)^{\varepsilon}$} \\
\hline$<6$ puntos & $36(19,4)$ & $3(10,3)$ & 0,237 \\
\hline 6 puntos & $56(30,3)$ & $13(44,8)$ & 0,119 \\
\hline$>6$ puntos & $93(50,3)$ & $13(44,8)$ & 0,586 \\
\hline
\end{tabular}

ALT: alanina aminotransferasa; AMA: anticuerpos antimitocondriales; ANA: anticuerpos antinucleares; ASMA: anticuerpos antimúsculo liso; AST: aspartato aminotransferasa; HAI: hepatitis autoinmune; IgG: inmunoglobulina G; RIC: rango intercuartil..

* Cardiopatía isquémica, insuficiencia cardíaca, enfermedad arterial periférica, accidente cerebrovascular.

TPorcentaje calculado sobre los pacientes con biopsia hepática en cada grupo.

${ }^{\text {E}}$ Según las recomendaciones del GIHA en Hennes EM et al. Hepatology. 2008 Jul;48(1):169-76. 
cirrosis al momento del diagnóstico en los pacientes mayores $(33,5 \%$ frente a $55.1 \%, p=0,024)$.

\section{Hallazgos de laboratorio}

No hubo diferencias en los parámetros bioquímicos ni en el perfil de autoanticuerpos entre los dos grupos (Tabla 1). El $82 \%$ de los pacientes en ambos grupos tuvo confirmación histológica del diagnóstico con hallazgos típicos o compatibles de HAI en más del $95 \%$ de los casos, y con un puntaje simplificado de HAI probable o definitivo en más del $80 \%$ de los pacientes. No hubo diferencias significativas en cuanto a la distribución del grado de fibrosis hepática por la escala METAVIR, sin embargo, hubo una mayor frecuencia de fibrosis $\mathrm{F} 4$ en el grupo de pacientes mayores $(39,9 \%$ frente a $58,3 \%, p=0,089$ ).

\section{Tratamiento, respuesta y evolución}

Como se describe en la Tabla 2, el tratamiento más frecuentemente recibido en ambos grupos fue la combina- ción de esteroide e inmunomodulador, sin encontrarse diferencias significativas entre los grupos. El grupo de los pacientes mayores tuvo una mayor frecuencia de remisión bioquímica con el tratamiento $(83,9 \%$ frente a $100 \%$, $p=0,022$ ), lo que permitió una mayor suspensión del tratamiento con esteroides, aunque sin ser significativa. Asimismo, los pacientes mayores tuvieron una menor frecuencia de recaídas durante el tratamiento (no significativa estadísticamente). En el 3,2 \% de los pacientes menores se pudo suspender por completo el tratamiento inmunosupresor. En ambos grupos, entre los pacientes no cirróticos, no hubo diferencias al momento del diagnóstico en cuanto al desarrollo de HAI durante el seguimiento. En total, se trasplantaron 13 pacientes, de los cuales solo uno fue del grupo de los pacientes mayores (una mujer diagnosticada a los 66 años, en estadio de cirrosis, en quien se indicó el trasplante hepático debido a las complicaciones relacionadas con la hipertensión portal). No hubo diferencias en cuanto al requerimiento de trasplante hepático, recurrencia de HAI postrasplante, retrasplante hepático y mortalidad.

Tabla 2. Características de tratamiento, respuesta al tratamiento y evolución en el tiempo

\begin{tabular}{lccc}
\hline & $\begin{array}{c}\text { Pacientes jóvenes } \\
<65 \text { años } \\
\mathrm{n}=185\end{array}$ & $\begin{array}{c}\text { Pacientes mayores } \\
\geq 65 \text { años } \\
\mathrm{n}=29\end{array}$ & Valor de $\boldsymbol{p}$ \\
\hline Tratamiento - $\mathrm{n}(\%)$ & $11(6)$ & $2(6,9)$ & 0,842 \\
$\quad$ Esteroide & $118(63,8)$ & $20(69)$ & 0,588 \\
Esteroide + inmunomodulador & $33(17,8)$ & $6(20,7)$ & 0,711 \\
Inmunomodulador, suspensión del esteroide & $6(3,2)$ & $0(0)$ & 0,327 \\
Suspensión del tratamiento & $10(5,4)$ & $1(3,4)$ & 0,657 \\
Ninguno & $7(3,8)$ & $0(0)$ & 0,284 \\
Sin dato & & & 0,022 \\
Respuesta al tratamiento - $\mathrm{n}(\%)^{*}$ & $141(83,9)$ & $28(100)$ & 0,053 \\
Remisión bioquímica & $20(11,9)$ & $0(0)$ & 0,357 \\
Remisión parcial & $5(3)$ & $0(0)$ & 0,561 \\
Falla terapéutica & $2(1,2)$ & $0(0)$ & 0,111 \\
Sin dato & $35(18,9)$ & $2(6,9)$ & 0,873 \\
Recaída - $\mathrm{n}(\%)^{*}$ & $18(14,6)$ & $2(16,6)$ & 0,522 \\
Desarrollo de cirrosis durante el seguimiento - $\mathrm{n}(\%)^{\pi}$ & $12(6,5)$ & $1(3,4)$ & 0,057 \\
Trasplante hepático - $\mathrm{n}(\%)$ & $2(16,6)$ & $1(100)$ & 0,764 \\
Recurrencia postrasplante - $\mathrm{n}(\%)^{\mathrm{n}}$ & $1(8,3)$ & $0(0)$ & 0,200 \\
Retrasplante - $\mathrm{n}(\%)$ & $10(5,4)$ & $0(0)$ & \\
Muerte - $\mathrm{n}(\%)$ & & & \\
\hline
\end{tabular}

*Porcentaje calculado sobre el total de los pacientes que recibieron tratamiento en cada grupo.

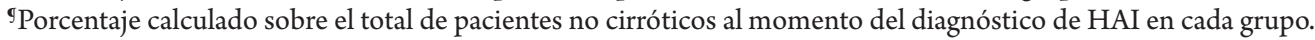

${ }^{\epsilon}$ Porcentaje calculado sobre el total de pacientes con trasplante hepático en cada grupo. 


\section{DISCUSIÓN}

A nivel mundial, los reportes sobre las características diferenciales de la HAI en personas mayores son escasos, de carácter retrospectivo y heterogéneos. Por ejemplo, para la definición de adulto mayor se han utilizado diferentes puntos de corte para la edad (a partir de los 60 o 65 años), y las poblaciones de pacientes más estudiadas son la norteamericana, la europea y la asiática (5), pero se desconocen datos en la población latinoamericana.

En total, existen 10 estudios que evalúan el comportamiento de la HAI en este grupo de pacientes, los cuales han sido recientemente analizados en una revisión sistemática de la literatura (5). El presente estudio es el primero que describe el comportamiento diferencial de la HAI en adultos latinoamericanos mayores, quienes constituyen un porcentaje no despreciable del total de pacientes con HAI: $12,9 \%$ (29 de 224) en este estudio, y el 10,4 \% (29 de 278), tomando como referencia la población de pacientes con HAI recientemente publicada por nuestro grupo (19), ambos porcentajes muy inferiores a los reportados globalmente $(24,8 \%)(5)$; sin embargo, dichas diferencias posiblemente tengan varias explicaciones: primero, el escaso número de pacientes reportados a nivel mundial (en total 264 pacientes adultos mayores con HAI); segundo, la heterogeneidad de las poblaciones estudiadas con diferentes criterios de inclusión y puntos de corte para la edad ( 3 estudios con punto de corte a partir de los 60 años y 7 estudios a partir de los 65 años) (5); y tercero, los criterios de inclusión y exclusión del presente estudio, ya que se utilizó el punto de corte de 65 años de edad, el cual es el más usado en los estudios reportados, la inclusión de algunos pacientes sin biopsia hepática $(20,9 \%)$ y con puntaje simplificado de HAI $<6$ puntos $(17,4 \%)$, en los cuales la respuesta al tratamiento permitió confirmar el diagnóstico de HAI $(2,17)$. Además, la exclusión de pacientes menores de 18 años, aquellos con síndromes de superposición HAI-CBP y HAI-CEP, HAI inducida por medicamentos y falla hepática aguda por HAI, en los cuales se ha descrito un curso y un pronóstico diferentes $(3,20-22)$.

Se corrobora el predominio de la afectación en mujeres en ambos grupos y el comportamiento bimodal en cuanto a la edad (2). Cabe resaltar que el $22 \%$ de los pacientes fue diagnosticado a partir de los 60 años, lo que refuerza la importancia de tener en cuenta a la HAI como una causa de hepatopatía en este grupo de edad. Este porcentaje fue similar a lo reportado en otros estudios $(20 \%)(4,8,9)$.

Aunque no hubo diferencias entre los grupos en cuanto a la forma de presentación clínica, es importante resaltar que casi una quinta parte de los pacientes eran asintomáticos, ya que se ha encontrado que hasta el $26 \%$ de los pacientes que no presentan síntomas están cirróticos al momento del diagnóstico de HAI (23). Se debe considerar a la HAI como una entidad para descartar ante la presencia de una alteración del perfil bioquímico-hepático en adultos, sin importar el grupo de edad $(2,11)$.

Como hallazgo relevante en este estudio, se encontró que los adultos mayores tuvieron un mayor grado de fibrosis hepática al momento del diagnóstico de HAI y, aunque esta diferencia no fue significativa en cuanto a la fibrosis F4 por biopsia hepática $(58,3 \%$ frente a $39.9 \%, p=0,089)$, sí fue significativa al evaluar la presencia de cirrosis por clínica (ascitis, circulación colateral, encefalopatía, ginecomastia, telangiectasias), pruebas analíticas (hipoalbuminemia, trombocitopenia, tiempos de coagulación prolongados), imagen y biopsia ( $55,1 \%$ frente a $33,5 \%, p=$ $0,0024)$, lo que concuerda con lo reportado en diferentes poblaciones a nivel mundial (5). Lo anterior sugiere un curso indolente de la HAI en personas mayores, el cual, como lo plantea Czaja (7), podría ser explicado por varias razones: el curso subclínico de la enfermedad, que puede llevar a un retraso en el diagnóstico $(10,11)$; la mayor prevalencia de comorbilidades, como hipertensión arterial, enfermedad cardiovascular, osteoporosis y neoplasias, que sugieran un diagnóstico alternativo y condicionen el uso de corticoesteroides para el tratamiento de HAI; además, una mayor frecuencia de comorbilidades autoinmunes en pacientes con HLA-DRB1 ${ }^{*} 04$, que pueden enmascarar las manifestaciones hepáticas (8). En este estudio, las diferencias significativas encontradas de una presencia mayor de hipertensión arterial, dislipidemia y enfermedad cardiovascular en la población mayor no impactó en cuanto al tipo de tratamiento recibido para la HAI, para el cual no se encontraron diferencias; tampoco se encontraron diferencias con respecto a las comorbilidades autoinmunes.

El envejecimiento conlleva una serie de cambios que alteran la homeostasis de la respuesta inmune (24), lo que puede influenciar en la presentación y el curso de enfermedades autoinmunes. Las más estudiadas son las enfermedades autoinmunes extrahepáticas clásicas, como el lupus eritematoso sistémico, la artritis reumatoide y el síndrome de Sjögren (24). Más allá del proceso de inmunosenescencia, se han encontrado cambios en la arquitectura de los órganos de la respuesta inmune, alteraciones en el balance entre factores proinflamatorios y antinflamatorios, y proapoptóticos y antiapoptóticos, que pueden modificar la respuesta inmune tanto humoral como celular $(24,26,27)$. En personas mayores, se reporta una mayor atrofia tímica con una disminución de la respuesta inmune mediada por los linfocitos $\mathrm{T}$ (27) y sin afectación de la respuesta humoral (28), lo que podría explicar que en la HAI existe una hiperglobulinemia y una respuesta al tratamiento inmunosupresor mayores (11) y una frecuencia de recaídas menor (5), estas dos últimas corroboradas en el presente estudio 
(remisión bioquímica $100 \%$ frente a 83,9\%, $p=0,022$ ). Las indicaciones y el esquema de tratamiento recomendados para la HAI en la población mayor no difieren de las de la población general (2); sin embargo, el tratamiento puede verse condicionado por una mayor presencia de comorbilidades y una mayor frecuencia del desarrollo de efectos adversos $(7,9)$. Cabe destacar que no encontramos diferencias en el desarrollo de efectos adversos relacionados con el tratamiento en nuestra población.

En este estudio nos encontramos con varias limitaciones: primero, las inherentes a un estudio retrospectivo, especialmente el sesgo de información, ya que se recolectaron los datos de la base de historia clínica electrónica del hospital; segundo,, se trata de un estudio de un solo centro, sin embargo, es un centro de referencia para enfermedades hepáticas a nivel nacional y cuenta con el número más grande de pacientes con HAI publicado a nivel de Latinoamérica (19); tercero, se incluyeron algunos pacientes sin biopsia hepática y con puntaje simplificado de $\mathrm{HAI}<6$, sin embargo, estos pacientes tuvieron respuesta al tratamiento farmacológico, lo que ayudó a corroborar el diagnóstico; cuarto, no se realizó la caracterización del HLA, del cual se han demostrado diferentes haplotipos (HLA-DQ2 y HLA-DR2) como factor de riesgo para HAI en la población latinoamericana (29) y en pacientes mayores (HLA-DR4 y HLA-DRB1*04) $(5,8)$. Por último, las diferencias en el tiempo de seguimiento de los pacientes, que fue menor en aquellos con $\geq 65$ años, también se han encontrado en los estudios más representativos de HAI en adultos mayores realizados por Al-Chalabi y colaboradores y Czaja y colaboradores $(4,8)$, que en nuestro estudio podrían explicarse debido a una mayor sensibilización en los últimos años en la búsqueda de HAI en los pacientes mayores, ya que el $72,4 \%$ de los pacientes fueron diagnosticados entre los años 2013 y 2016.

Como fortalezas destacamos el número de pacientes incluidos, ya que es uno de los más grandes que evalúa el comportamiento de HAI en este grupo de pacientes (5); el seguimiento en el tiempo, que permitió conocer el comportamiento diferencial de la HAI según el grupo de edad; el porcentaje de pacientes ( $82 \%$ ) con diagnóstico confirmado con biopsia hepática; y la descripción detalladamente de la forma de presentación clínica y la evaluación de la respuesta al tratamiento, ya que estos aspectos no fueron evaluados en algunos de los estudios sobre HAI en adultos mayores (5).

En conclusión, la HAI afecta a los adultos de todas las edades, aunque es habitual en aquellos con una edad $\geq 65$ años, los cuales tienen una mayor frecuencia de cirrosis al momento del diagnóstico. Se debe tener en cuenta a la HAI como una causa de hepatopatía en este grupo de pacientes, con el fin de establecer un diagnóstico y un tratamiento opor- tunos, ya que los pacientes presentan una mejor respuesta al tratamiento sin una mayor frecuencia de efectos adversos.

\section{Conflicto de intereses}

Los autores manifiestan que no existe ningún conflicto de intereses.

\section{Fuente de financiación}

Propia.

\section{REFERENCIAS}

1. Waldeström J. Leber, Blut proteine und Nahrungseiweiss. DischZ Verdan Stoff'Wechselkr. 1950;15:113-6.

2. European Association for the Study of the Liver. EASL Clinical Practice Guidelines: Autoimmune hepatitis. J Hepatol. 2015 Oct;63(4):971-1004. https://doi. org/10.1016/j.jhep.2015.06.030. Epub 2015 Sep 1. Erratum in: J Hepatol. 2015 Dec;63(6):1543-4.

3. Mieli-Vergani G, Vergani D. Autoimmune paediatric liver disease. World J Gastroenterol. 2008 Jun 7;14(21):3360-7. https://doi.org/10.3748/wjg.14.3360.

4. Al-Chalabi T, Boccato S, Portmann BC, McFarlane IG, Heneghan MA. Autoimmune hepatitis (AIH) in the elderly: a systematic retrospective analysis of a large group of consecutive patients with definite AIH followed at a tertiary referral centre. J Hepatol. 2006 Oct;45(4):575-83. https://doi. org/10.1016/j.jhep.2006.04.007.

5. Chen J, Eslick GD, Weltman M. Systematic review with meta-analysis: clinical manifestations and management of autoimmune hepatitis in the elderly. Aliment Pharmacol Ther. 2014 Jan;39(2):117-24. https://doi.org/10.1111/ apt.12563.

6. Mieli-Vergani G, Vergani D, Czaja AJ, Manns MP, Krawitt EL, Vierling JM, et al. Autoimmune hepatitis. Nat Rev Dis Primers. 2018 Apr 12;4:18017. https://doi.org/10.1038/ nrdp.2018.17.

7. Czaja AJ. Special clinical challenges in autoimmune hepatitis: the elderly, males, pregnancy, mild disease, fulminant onset, and nonwhite patients. Semin Liver Dis. 2009 Aug;29(3):31530. https://doi.org/10.1055/s-0029-1233530.

8. Czaja AJ, Carpenter HA. Distinctive clinical phenotype and treatment outcome of type 1 autoimmune hepatitis in the elderly. Hepatology. 2006 Mar;43(3):532-8. https://doi. org/10.1002/hep.21074.

9. Schramm C, Kanzler S, zum Büschenfelde KH, Galle PR, Lohse AW. Autoimmune hepatitis in the elderly. Am J Gastroenterol. 2001 May;96(5):1587-91. https://doi. org/10.1016/S0002-9270(01)02345-0.

10. Granito A, Muratori L, Pappas G, Muratori P, Ferri S, Cassani $\mathrm{F}$, et al. Clinical features of type 1 autoimmune hepatitis in elderly Italian patients. Aliment Pharmacol Ther. 2005 
May 15;21(10):1273-7. https://doi.org/10.1111/j.13652036.2005.02488.x.

11. Czaja AJ. Clinical features, differential diagnosis and treatment of autoimmune hepatitis in the elderly. Drugs Aging. 2008;25(3):219-39. https://doi.org/10.2165/00002512200825030-00005.

12. Verslype C, George C, Buchel E, Nevens F, van Steenbergen W, Fevery J. Diagnosis and treatment of autoimmune hepatitis at age 65 and older. Aliment Pharmacol Ther. 2005 Mar 15;21(6):6959. https://doi.org/10.1111/j.1365-2036.2005.02403.x.

13. Parker DR, Kingham JG. Type I autoimmune hepatitis is primarily a disease of later life. QJM. 1997 Apr;90(4):28996. https://doi.org/10.1093/qjmed/90.4.289.

14. Newton JL, Burt AD, Park JB, Mathew J, Bassendine MF, James OF. Autoimmune hepatitis in older patients. Age Ageing. 1997 Nov;26(6):441-4. https://doi.org/10.1093/ ageing/26.6.441.

15. Miyake Y, Iwasaki Y, Takaki A, Kobashi H, Sakaguchi K, Shiratori Y. Clinical features of Japanese elderly patients with type 1 autoimmune hepatitis. Intern Med.2007;46(24):19459. https://doi.org/10.2169/internalmedicine.46.0420.

16. Hennes EM, Zeniya M, Czaja AJ, Parés A, Dalekos GN, Krawitt EL, et al. Simplified criteria for the diagnosis of autoimmune hepatitis. Hepatology. $2008 \mathrm{Jul}$;48(1):169-76. https://doi.org/10.1002/hep.22322.

17. Alvarez F, Berg PA, Bianchi FB, Bianchi L, Burroughs AK, Cancado EL, et al. International Autoimmune Hepatitis Group Report: review of criteria for diagnosis of autoimmune hepatitis. J Hepatol. 1999 Nov;31(5):929-38. https:// doi.org/10.1016/S0168-8278(99)80297-9.

18. Vandenbroucke JP, von Elm E, Altman DG, Gøtzsche PC, Mulrow CD, Pocock SJ, et al. Strengthening the Reporting of Observational Studies in Epidemiology (STROBE): explanation and elaboration. Ann Intern Med. 2007 Oct 16;147(8):W163-94. https://doi.org/10.7326/0003-4819147-8-200710160-00010-w1.

19. Díaz-Ramírez GS, Marín-Zuluaga JI, Donado-Gómez JH, Muñoz-Maya O, Santos-Sánchez Ó, Restrepo-Gutiérrez JC. Characterization of patients with autoimmune hepatitis at an university hospital in Medellín-Colombia: cohort study. Gastroenterol Hepatol. 2018 Feb;41(2):87-96. https://doi. org/10.1016/j.gastrohep.2017.09.003.
20. Czaja AJ. Diagnosis and management of the overlap syndromes of autoimmune hepatitis. Can J Gastroenterol. 2013 Jul;27(7):417-23. https://doi.org/10.1155/2013/198070.

21. Björnsson E, Talwalkar J, Treeprasertsuk S, Kamath PS, Takahashi N, Sanderson S, et al. Drug-induced autoimmune hepatitis: clinical characteristics and prognosis. Hepatology. 2010 Jun;51(6):2040-8. https://doi.org/10.1002/hep.23588.

22. Kessler WR, Cummings OW, Eckert G, Chalasani N, Lumeng L, Kwo PY. Fulminant hepatic failure as the initial presentation of acute autoimmune hepatitis. Clin Gastroenterol Hepatol. 2004 Jul;2(7):625-31. https://doi. org/10.1016/S1542-3565(04)00246-0.

23. Feld JJ, Dinh H, Arenovich T, Marcus VA, Wanless IR, Heathcote EJ. Autoimmune hepatitis: effect of symptoms and cirrhosis on natural history and outcome. Hepatology. 2005 Jul;42(1):53-62. https://doi.org/10.1002/hep.20732.

24. Watad A, Bragazzi NL, Adawi M, Amital H, Toubi E, Porat BS, et al. Autoimmunity in the Elderly: Insights from Basic Science and Clinics - A Mini-Review. Gerontology. 2017;63(6):515523. https://doi.org/10.1159/000478012.

25. Amador-Patarroyo MJ, Rodriguez-Rodriguez A, MontoyaOrtiz G. How does age at onset influence the outcome of autoimmunediseases?AutoimmuneDis.2012;2012:251730. https://doi.org/10.1155/2012/251730.

26. Schwab R, Russo C, Weksler ME. Altered major histocompatibility complex-restricted antigen recognition by $\mathrm{T}$ cells from elderly humans. Eur J Immunol. 1992 Nov;22(11):2989-93. https://doi.org/10.1002/eji.1830221134.

27. De Paoli P, Battistin S, Santini GF. Age-related changes in human lymphocyte subsets: progressive reduction of the CD4 CD45R (suppressor inducer) population. Clin Immunol Immunopathol. 1988 Sep;48(3):290-6. https:// doi.org/10.1016/0090-1229(88)90022-0.

28. Ben-YehudaA,WekslerME.Immunesenescence:mechanisms and clinical implications. Cancer Invest. 1992;10(6):525-31. https://doi.org/10.3109/07357909209024815.

29. Duarte-Rey C, Pardo AL, Rodríguez-Velosa Y, Mantilla RD, Anaya JM, Rojas-Villarraga A. HLA class II association with autoimmune hepatitis in Latin America: a meta-analysis. Autoimmun Rev. 2009 Feb;8(4):325-31. https://doi. org/10.1016/j.autrev.2008.11.005. 